\title{
Conceptualization and Implementation of a Microscopic Pedestrian Simulation Platform
}

\author{
Edgar F. Esteves, Rosaldo J. F. Rossetti, Paulo A. F. Ferreira, Eugénio C. Oliveira \\ Artificial Intelligence and Computer Science Laboratory (LIACC) \\ Faculty of Engineering, University of Porto (FEUP) \\ Rua Dr. Roberto Frias, S/N • 4200-465 Porto • Portugal \\ $\mathrm{T:}(+351) 225081566 \mathrm{~F}:(+351) 225081440$ \\ \{edgar.esteves, rossetti, paff, eco\}@fe.up.pt
}

\begin{abstract}
This work reports on our first steps toward the implementation of a platform for pedestrian simulation in multimodal exchange interfaces. Whereas most approaches in pedestrian simulation are aimed at studying interactions among people using a common environment with different purposes and under different mobility restrictions, our research is focused on how those interactions will ultimately affect transportation operations at multimodal stations. This paper starts with a general overview of pedestrian simulation, focusing on the microscopic representation of individuals and their interoperability that will be the basis for the system architecture briefly presented.
\end{abstract}

\section{Keywords}

Microscopic pedestrian modelling and simulation, multimodal transport exchange interfaces, multi-agent systems, OpenSteer.

\section{INTRODUCTION}

The prediction of the influence of urban planning on people comfort and mobility, both inside public buildings and open areas is becoming a very important process. Several studies have then been carried out as an attempt to predict these influences, either by means of traditional multi-purpose simulation tools or by the development of modern pedestrian simulators. While most of those models are rather oriented to understand how space will affect crowd flow, we focus on the other way round trying to realise how a better understanding of crowd behaviour can improve space design, especially in public transport multimodal exchange interfaces.

Much knowledge on pedestrian traffic systems comes from empirical studies, discussed in greater detail in [1][2]. According to [3] pedestrian studies can be divided into pedestrian data collection and pedestrian analysis. While data collection focuses on the observation pedestrian characteristics, pedestrian analysis is concerned with interpretation of collected data both to understand observed situations and to plan new facilities. Intuitively, these studies have an analogy to vehicular traffic

Permission to make digital or hard copies of all or part of this work for personal or classroom use is granted without fee provided that copies are not made or distributed for profit or commercial advantage and that copies bear this notice and the full citation on the first page. To copy otherwise, or republish, to post on servers or to redistribute to lists, requires prior specific permission and/or a fee.

SAC'09, March 8-12, 2009, Honolulu, Hawaii, U.S.A.

Copyright 2009 ACM 978-1-60558-166-8/09/03 ...\$5.00. analysis since they can also be divided into two categories, namely macroscopic and microscopic models. According to Helbing and colleagues [2], microscopic models may follow different approaches, such as cellular automata, physical forces, queuing networks and AI-based techniques.

In cellular-based models, pedestrians are simulated as a particle in a cell. The environment is represented by a grid of cells and movement is modelled as cell switching. The disadvantage of this model is the high discretisation of space, whereas its simplicity reduces computational time. In contrast, physical force models can predict the precise position of pedestrians. There are two approaches to this model, namely magnetic forces and social forces. The former applies magnetic fields principia, whereas the latter combines the basis of a cost-benefit cellular model and magnetic forces to compose pedestrians' social forces. The queuing network model is often used to analyse evacuation situations. In this approach, each room is a node and doors between them are links. Pedestrians depart from one node, queue in a link and arrive at another node.

AI-based approaches actually can be seen as complementary to the previous ones, bringing new features to pedestrian simulation. The main contribution given to other models is the improvement of space searching queries and pedestrian decision making, through the use of algorithms such as $\mathrm{A}^{*}$ and Dijkstra. More recently, Distributed Artificial Intelligence techniques, such as multi-agent systems and agent-based simulation, have been greatly applied to pedestrian simulation with promising success. In agent-based models we can identify different options regarding how sophisticated one agent is to be implemented. Very simple (reactive) agents can be used to simulate a whole population of pedestrians and still can produce a good approximation to the actual overall system behaviour. This allows a great number of agents to be simulated. Another option is to model each agent in detail, with more complex (cognitive) behaviour. Albeit appropriate to represent human decision-making, they are subject to great criticism. We intend to combine both options in an adaptable agent architecture for pedestrian simulation.

\section{SYSTEM ARCHITECTURE}

After a thoroughly literature review, we conceptualized a system capable of performing microscopic pedestrian simulation of most common scenarios. Nevertheless, its modularity also allows us to use it in other kinds of simulation, such as pedestrian simulation under emergency conditions or even vehicular traffic simulation. The system architecture has two distinct modules, namely the Simulation Editor (SE) and the Simulation Engine Controller 
(SEC), which contain their own components. SE is responsible for scenario definition, in which elements such as walls, lines, points of attraction, and equipments are set up, as well as are various other parameters such as entry and exit flows and occupation of components. A Network Edition component adds to SE a set of features that allows it to create an entire scene from scratch. The specification of scene objects is performed through a graphical user interface (GUI), allowing the user to set up all related parameters. To ease understanding, imagine a train station scenario. After creating a simple polygon, it is possible to set it up as a room, a bar, a ticket office or even as an interchange zone, and similarly a line might be set up as a wall or movement delimiter. It is also possible to create a predefined set of equipments, such as ATM, elevators and escalators. The Moveables Configuration component allows us to set up all parameters of crowd characteristics, such as flow entering in and exiting from the study scenario. Individual entities' maximum and minimum speeds, centre of mass and behavioural rules are also defined through this component.

On the other hand, SEC was conceptualized on the basis of microscopic simulation using AI-based modelling approaches. The Network Layer Manager component provides information about simulation scenarios, which might be tailored accordingly so as to be used by different purpose simulation plug-ins to be attached to the system architecture. Simulation plug-ins are handled by the Plug-in Layer Manager component, whereas another important component, the Visualization Layer Manager, provides the 3D Visualization Module with scenario objects rules to define and change levels of visualization details (e.g. show or hide walls). The Moveable Physics component carries out all vector operations to handle the $3 \mathrm{D}$ space; these calculations are crucial for the steering behaviours provided by the Moveable Steering Behaviour component. For basic human movement behaviours, it is essential that this component contains at least steering behaviours such as seek, obstacle avoidance, wander, path following, unaligned collision avoidance, cohesion and alignment. Readers are referred to [4] for a detailed explanation on how to implement these behaviours.

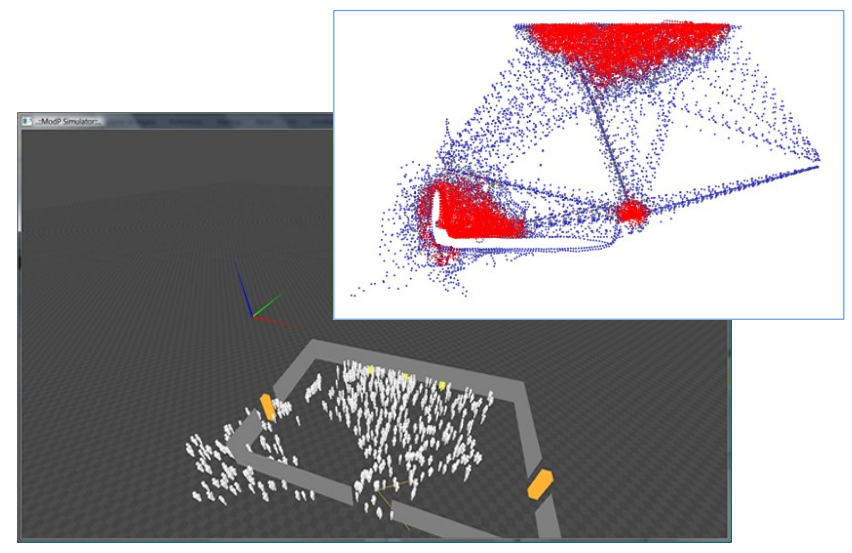

Figure 1. Snapshots of the framework GUI.

In our approach, a scenario is composed of at least one Level. These Level objects do not have a rigid structure and represent areas where pedestrians circulate to accomplish some tasks; in other words, a level can be a room or a floor with rooms. Figure 1 depicts snapshots of the framework GUI, where a level can be observed and analysed (e.g. via density graphs). In train station scenarios, for instance, if a traveller wants to have a snack or to buy a ticket he/she will search for restaurants or ticket offices nearby, which can be rooms on the current level. These rooms are delimited by walls and have a set of components inside. Components are centred in three-dimensional points and have orientation vectors. Walls and MovementDelimiters are lines that constrain pedestrian movement and safeguard scenario rules. Pedestrians enter and exit the scenario through drain and sources, whereas connector objects are meant not only to provide a connection function between levels but also to provide semantics regarding the level to which they are connected. For example, when a traveller in a train station enters the station he/she does it through entry doors. At the moment he/she passes the door, the traveller reads information about the environment layout, such as "Ticket Offices Area Left", "Restaurant Area 1st Floor", "Interchange Platform Front", among other useful information. Such information is then used in pedestrians' decision-making.

\section{CONCLUSION}

We have implemented a first prototype to underlie the development of our system architecture. The use of the OpenSteer [5] library to support a detailed representation of pedestrian dynamics has proven to be very appropriate and easy to adapt to our simulation framework. As for the environment representation, it is important to retain the relevance of object abstractions if the major concern is to ensure the reuse of the implementation of the steering behaviours and of the pedestrians' decision processes in different scenarios. The very next step in this research work is the development of a BDI architecture [6] to underlie the implementation of pedestrian agents. We are also aiming at the definition of a validation and calibration methodology, so as to test the approach in real scenarios.

\section{ACKNOWLEDGMENTS}

Our sincere thanks to Oana Santos and Vera Ferreira, from TRENMO Engenharia Ltd., for their invaluable contributions. This work has been partially supported by ADI/RCM, Portugal.

\section{REFERENCES}

[1] Helbing, D., Molnár, P., Farkas, I. J., Bolay, K. (2001) Selforganizing pedestrian movement. Environment and Planning B: Planning and Design, 28(3)361-383.

[2] Helbing, D., Farkas, I. J., Molnár, P., Vicsek, T. (2002) Simulation of Pedestrian Crowds in Normal and Evacuation Situations. In Pedestrian and Evacuation Dynamics, 21-58.

[3] Teknomo, K. (2002) Microscopic Pedestrian Flow Characteristics: development of an Image Processing Data Collection and Simulation Model. Ph.D. Thesis, Tohoku University, Japan.

[4] Reynolds, C.W. (1999) Steering behaviors for autonomous characters. In Proc. Game Developers Conference, 763-782.

[5] Reynolds, C. W. (2004) OpenSteer Documentation. [Online] http://opensteer.sourceforge.net/index.html (last accessed Oct. 25, 2008).

[6] Bordini, R. H., Hübner, J. F. (2006) BDI agent programming in AgentSpeak using Jason. In Proc Computational Logic in Multi-Agent Systems (LNCS 3900). 143-164. 